\title{
Non-contact mapping to guide catheter ablation of untolerated ventricular tachycardia
}

\author{
P. Della Bella ${ }^{1}$, A. Pappalardo ${ }^{2}$, S. Riva ${ }^{1}$, C. Tondo ${ }^{1}$, G. Fassini ${ }^{1}$ and N. Trevisi ${ }^{1}$ \\ ${ }^{1}$ Institute of Cardiology, University of Milan, IRCCS, Centro Cardiologico 'Fondazione I. Monzino', Milan, and \\ ${ }^{2}$ Department of Heart Sciences, Ospedale S. Camillo, Rome, Italy
}

\begin{abstract}
Aims The role of a novel non-contact mapping system (ESI 3000, Endocardial Solutions) to guide radiofrequency catheter ablation of untolerated ventricular tachycardia was investigated in 17 patients; 11 with prior myocardial infarction, three with arrhythmogenic right ventricular dysplasia, and three with idiopathic dilated cardiomyopathy.
\end{abstract}

Methods Twenty-seven monomorphic ventricular tachycardias were induced (mean cycle $320 \pm 60 \mathrm{~ms}$, range 230 $450 \mathrm{~ms}$ ), mapped for 15-20 s, and terminated by overdrive pacing or DC shock. Off-line analysis of isopotential activation mapping was performed to identify the diastolic pathway and/or the exit point of the ventricular tachycardia reentry circuit. Radiofrequency current was applied to create a line of block across the diastolic pathway or around the exit point.

Results All 27 ventricular tachycardias were mapped with the non-contact system. The endocardial exit point ( $-7 \pm 15 \mathrm{~ms}$ before QRS onset) was defined in 21/21 postinfarction ventricular tachycardias, in $3 / 3$ arrhythmogenic right ventricular dysplasia and in $1 / 3$ idiopathic dilated cardiomyopathy ventricular tachycardias, respectively. The diastolic pathway (earliest endocardial diastolic activity: $-65 \pm 49 \mathrm{~ms}$ before QRS onset) was identified in $17 / 21$ postinfarction ventricular tachycardias, in $1 / 3$ arrhythmogenic right ventricular dysplasia and in $1 / 3$ idiopathic dilated cardiomyopathy ventricular tachycardias, respectively. Catheter ablation was performed in $25 / 27$ ventricular tachycardias $(93 \%)$ in $15 / 17$ patients $(88 \%)$ : $16 / 25$ ventricular tachycardias $(64 \%)$ were successfully ablated in $10 / 17$ patients $(59 \%)$. Catheter ablation was not performed in two patients or proved unsuccessful in five patients. At a follow-up of $15 \pm 5$ months, there was no recurrence of documented ventricular tachycardia in all 10 patients with successful catheter ablation; in two of them a previously non-documented ventricular tachycardia occurred. A high recurrence of ventricular tachycardia was observed in patients with a failed procedure (5/7: 71\%). No major complication or death occurred.

Conclusions Non-contact mapping can be effectively used to map and guide radiofrequency catheter ablation of untolerated ventricular tachycardias. Given the favourable acute and clinical long-term results, this approach proves to be more effective in patients with postinfarction ventricular tachycardias, in comparison to patients with arrhythmogenic right ventricular dysplasia and idiopathic dilated cardiomyopathy.

(Eur Heart J, 2002; 23: 742-752, doi:10.1053/euhj.2001. 2939)

(C) 2001 The European Society of Cardiology

Key Words: Non-contact mapping, ventricular tachycardia, radiofrequency catheter ablation.

See page 696 doi:10.1053/euhj.2001.3023 for the Editorial comment on this article

\section{Introduction}

Radiofrequency catheter ablation of ventricular tachycardia requires careful endocardial mapping combined with pacing manoeuvres to localize the area of slow

Received 14 March 2001, accepted 25 July 2001, and published online 27 November 2001.

Correspondence: Paolo Della Bella, MD, Institute of Cardiology, University of Milan, Centro Cardiologico, IRCCS, Via Parea, 4-20138 Milano, Italy. conduction critical for the maintenance of reentry ${ }^{[1-8]}$. This time-consuming procedure can be limited by haemodynamic intolerance to the tachycardia, thus limiting the overall possibility of catheter ablation techniques for ventricular tachycardia ${ }^{[9,10]}$.

A non-contact mapping technique (ESI 3000, Endocardial Solutions) has been recently introduced. The system is able to detect far field unipolar endocardial electrograms, generated at the endocardial surface, and to generate an isopotential activation map on a virtually reconstructed endocardium. Since it allows the analysis 
of a complete map from one tachycardia beat, the system can be used to study the pattern of endocardial activation of unstable arrhythmias and to guide catheter ablation.

A good correlation between contact and reconstructed endocardial electrograms both during sinus rhythm and ventricular tachycardia has been shown in previous studies $^{[11-14]}$; subsequently the feasibility of radiofrequency catheter ablation of human ventricular tachycardia, guided by non-contact mapping, has been demonstrated ${ }^{[15-17]}$.

In this study, we have prospectively investigated the use of the non-contact mapping system to guide radiofrequency catheter ablation of haemodynamically untolerated ventricular tachycardia.

\section{Methods}

\section{Inclusion criteria}

Patients were included with documented haemodynamically untolerated ventricular tachycardia (syncope or marked hypotension), unresponsive to antiarrhythmic drugs, including full dose amiodarone, in whom electrophysiological evaluation was required. Patients were considered eligible regardless of the presence of an implantable cardioverter-defibrillator (ICD).

During the electrophysiological study, the haemodynamic intolerance to an induced ventricular tachycardia was further demonstrated by the direct recording of arterial pressure. A ventricular tachycardia was defined as untolerated if the mean arterial pressure was less than $50 \mathrm{mmHg}$ at $30 \mathrm{~s}$ after induction and/or if interruption by overdrive pacing or cardioversion was required by development of pulmonary congestion or by loss of consciousness.

\section{Exclusion criteria}

Patients were not studied if they had: coronary artery disease and left ventricular aneurysm when intraoperative mapping-guided surgical intervention plus revascularization were considered; induced polymorphic ventricular tachycardia or ventricular tachycardia degenerating into ventricular fibrillation, documented at a previous electrophysiological study; presence of left ventricular fresh thrombus assessed by transthoracic echocardiogram; documented occlusive arterial disease of the iliofemoral tree. Patients with clinical or inducible sustained monomorphic ventricular tachycardia that was haemodynamically tolerated, were not included in the study.

\section{Patients}

From July 1988 to March 2000, 22 patients were referred for electrophysiological evaluation of haemodynamically untolerated ventricular tachycardia and were considered eligible for a non-contact mapping and radiofrequency catheter ablation procedure. Five patients were excluded because of the aforementioned criteria (two with confirmed iliofemoral vascular disease, two because of a coexisting surgical indication, one for inconsistent ventricular tachycardia inducibility). Seventeen patients were included in the study: 16 male, one female, mean age $55 \cdot 7 \pm 18.9$ years (range $24-75$ years); 11 patients had a previous myocardial infarction, arrhythmogenic right ventricular dysplasia was present in three and idiopathic dilated cardiomyopathy in three. Mean left ventricular ejection fraction was $40 \pm 10 \cdot 9 \%$.

All patients were being treated with antiarrhythmic drugs (15 with amiodarone, two with sotalol) and four (two with a previous myocardial infarction and two with arrhythmogenic right ventricular dysplasia) had an ICD previously implanted and were referred for excessive ICD therapy $(10 \pm 2.5$ shocks/month). Other patient characteristics are reported in Table 1.

\section{Non-contact mapping system}

Technical details of the commercially available system (Endocardial Solutions ESI 3000) have been described elsewhere $e^{[11-17]}$. Briefly, a $9 \mathrm{~F}$ catheter is percutaneously introduced in the ventricular cavity and connected to a multichannel recorder and amplifier system to acquire more than 3000 non-contact unipolar electrograms that are subsequently processed by a Silicon Graphics workstation. Through the processing of the recorded electrograms, the system generates a colour coded isopotential map that projects over the virtual endocardium the activation and repolarization wavefront of the whole cycle. It is possible, at any given interval of the cycle, to display the virtual unipolar endocardial electrograms from a chosen site of the endocardial surface. This allows analysis of the following relevant parameters of the ventricular tachycardia reentry circuit:

(1) Exit point: the site where a QS unipolar EGM morphology is recorded, which, on the map, corresponds to the site of earliest activation followed by a rapid activation front, spreading to the surrounding healthy endocardium ${ }^{[15,16]}$. Although this generally coincides with the surface QRS onset, sometimes it is found to precede it by $10-30 \mathrm{~ms}^{[18-20]}$.

(2) Diastolic pathway: a defined region of endocardium, usually bordered by scar tissue where diastolic electrical activity, preceding the exit point, is recorded over a variable amount of the diastolic interval.

(3) Diastolic interval: the part of the ventricular tachycardia cycle between the end of a QRS complex and the onset of the next one.

\section{Mapping and ablation procedure}

An informed written consent was obtained by all patients before the study. All patients were studied in the 


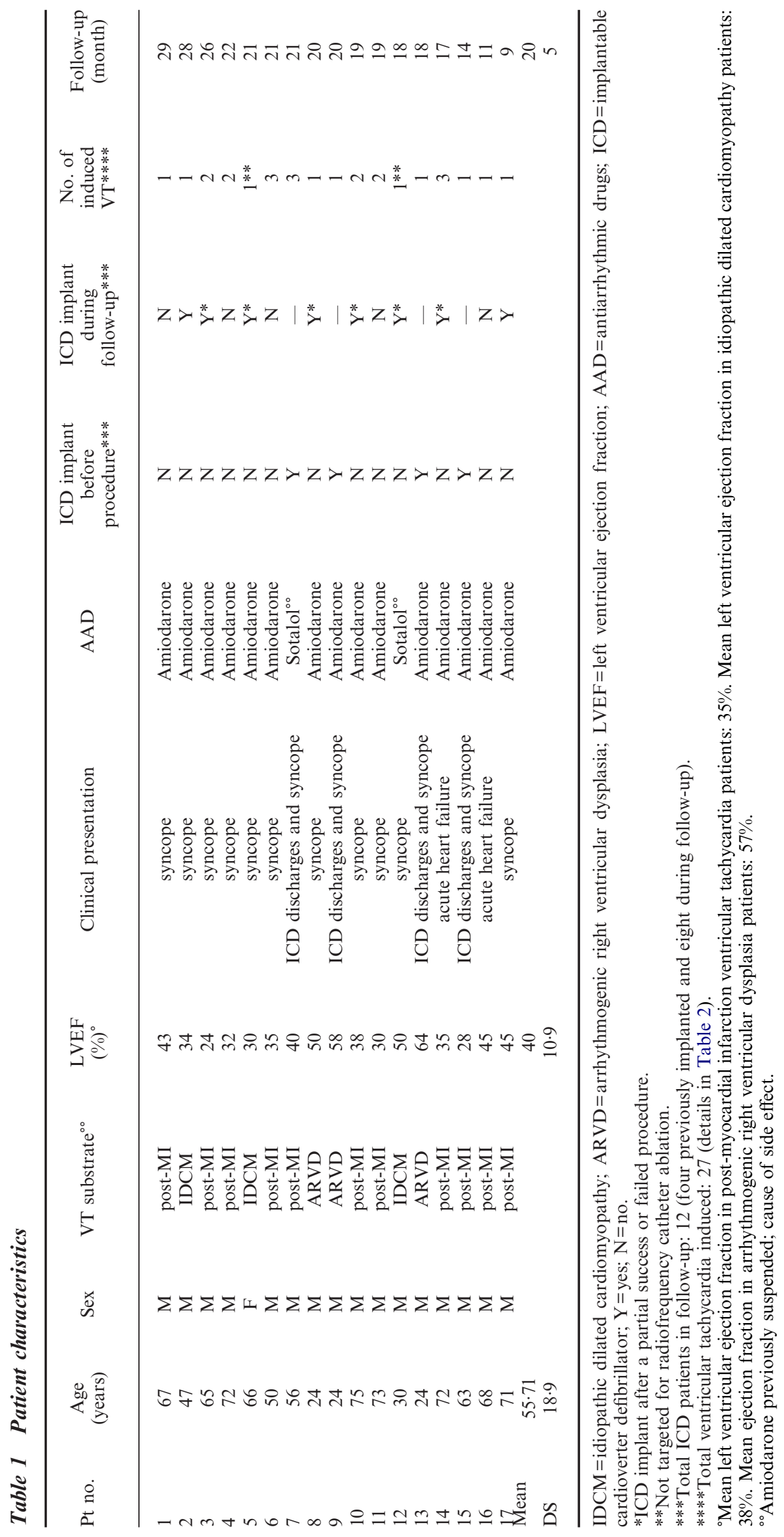




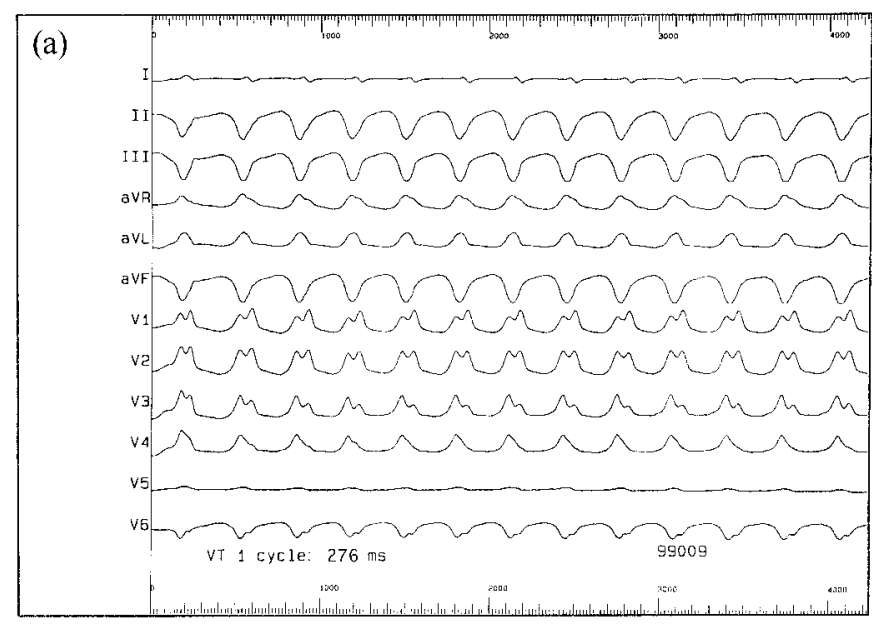

Figure 1 (a).

postabsorptive state. A standard quadripolar catheter was advanced to the right ventricular apex and a standard, deflectable, mapping/ablation $4 \mathrm{~mm}$-tip catheter was placed in the left ventricle by a retrograde transaortic route or in the right ventricle in patients with ventricular tachycardia related to arrhythmogenic right ventricular dysplasia. Systemic anticoagulation was obtained with 10000 IU heparin i.v. bolus followed by a continuous infusion to maintain an activated clotting time $>300 \mathrm{~s}$. By a retrograde-transaortic or caval-right atrial route, the multielectrode catheter was advanced to the left ventricular apex or to the outflow tract of the right ventricle over a 0.032 inch J-tipped guidewire. Right anterior oblique (RAO) and left anterior oblique (LAO) views were used to assess the correct position of the balloon catheter, placed parallel to the long axis and with the pigtail end as close as possible to the left ventricular apex or to the pulmonary valve in patients undergoing right ventricular procedures. Then the guidewire was withdrawn and the balloon inflated with a contrast-saline mixture.

$\mathrm{O}_{2}$ saturation and systemic arterial blood pressure were monitored continuously.

Contact catheter data and the surface ECG were recorded simultaneously on a conventional electrophysiology system (Bard EP Labsystem) and the non-contact mapping system.

The construction of the virtual endocardium model was preliminarily obtained during sinus rhythm, by handling the ablation catheter along the endocardial surface to collect a series of geometric points. Programmed ventricular stimulation using a standard protocol was undertaken to induce the documented ventricular tachycardia. A 15-20 s segment of any induced ventricular tachycardia was recorded with the non-contact system and subsequently terminated by either overdrive pacing or DC shock. Once haemodynamic stability was regained during sinus rhythm, off-line analysis was performed. Starting from the beginning of the QRS, localization of the earliest endocardial activation, guided by the colour coded isopotential map, was assessed. Projection of the virtual endocardial electrograms over this area was performed at different high-pass filter settings $(1,2,4,8,16$ and $32 \mathrm{~Hz})$ in order to avoid misinterpretation with repolarization waveforms. To identify the diastolic pathway and/or the exit point of the ventricular tachycardia reentry circuit, a time cursor was moved backwards in time until no endocardial activity could be observed on the isopotential map, even at the highest level of amplification and sensitivity. This process was repeated to reconfirm the identity of the diastolic pathway and/or exit point. Catheter ablation was performed by delivering $1 \mathrm{~min}$ pulses of radiofrequency current under thermal control $\left(60^{\circ}, 50 \mathrm{~W}\right)$. Whenever a diastolic pathway could be identified, a series of radiofrequency lesions were delivered, in series, to create a line of block across the diastolic pathway (Figs 1 and 2). If only the exit point was clearly mapped, a series of lesions was designed to encircle the target site in an attempt to create an area of block surrounding the exit point.

\section{End-points}

After termination of the designed ablation protocol, the effectiveness of the procedure was tested by repeating programmed stimulation (up to three extrastimuli). A successful procedure was defined as the prevention of the inducibility of any ventricular tachycardia, including the documented arrhythmia. If a different ventricular tachycardia was induced, a new mapping and ablation procedure was undertaken and the efficacy was subsequently tested. Prevention of the documented ventricular tachycardia with persistent inducibility of one or more different ventricular tachycardias was defined as a partial success.

An ICD was implanted following the procedure in patients with failure or partial success. Patients were discharged after 3 days of continuous electrocardiographic monitoring and scheduled for an outpatient visit at 1 month and every 3 months. Patients were instructed 

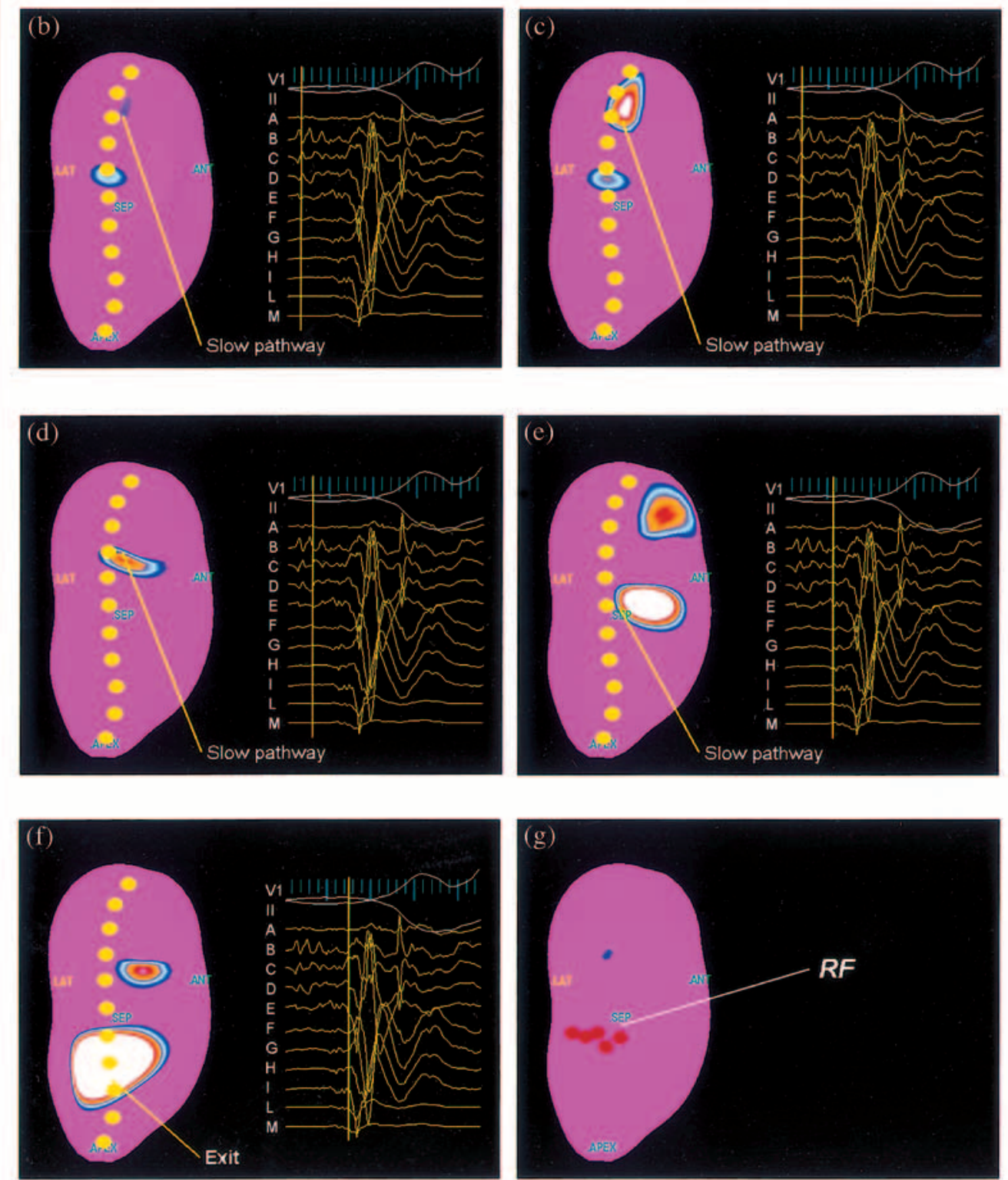

Figure 1 (b)-(g).

Figure 1 Panel a: 12 lead ECG of sustained untolerated ventricular tachycardia in patient no. 4 with an old anteroseptal myocardial infarction. Ventricular tachycardia cycle length: $276 \mathrm{~ms}$. Panels b-g: A single ventricular tachycardia beat is shown. For each panel, the reconstruction of the left ventricle endocardium is shown on the left in purple as seen from the septal wall; activated myocardium is shown in colours from white to blue. Selected unipolar virtual electrograms (A to $M$ ) at different timings during a ventricular tachycardia beat are shown on the right. Diastolic fragmented activity is demonstrated at electrogram A to $G$ (slow pathway), unipolar QS electrograms from I to M (exit site). A line of radiofrequency lesions (red dot, panel g) was drawn to interrupt the diastolic pathway, before the exit site.

to contact the referring physician in case of ICD discharge or any symptom related to ventricular tachyarrhythmias.

\section{Statistics}

Continuous data are presented as a mean and SD. Statistical analysis was performed using chi-square or two-tailed Student's $t$-test. A $P$ value $\leq$ to 0.05 was chosen as statistically significant.

\section{Results}

Twenty-seven sustained monomorphic ventricular tachycardias were induced: 21 in patients with 


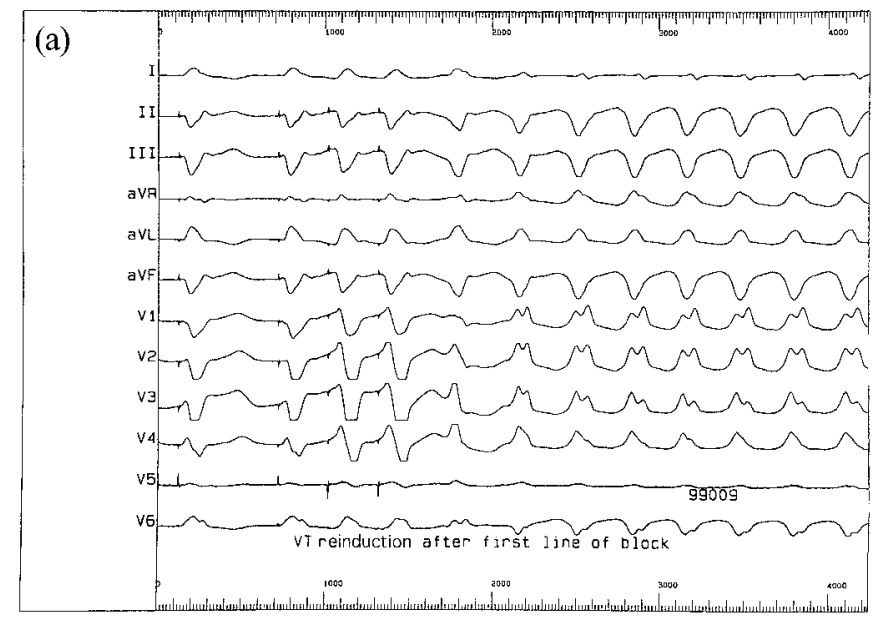

Figure 2 (a).

previous myocardial infarction, three in patients with arrhythmogenic right ventricular dysplasia, three in patients with idiopathic dilated cardiomyopathy. The mean ventricular tachycardia cycle length was $320.63 \pm 60.66 \mathrm{~ms}$ (range $230-450 \mathrm{~ms}$ ). All induced ventricular tachycardias were untolerated as previously defined and needed interruption by overdrive or external cardioversion. Procedural data are reported in Table 2.

\section{Mapping}

All untolerated ventricular tachycardias were mapped with the non-contact system that identified an endocardial ventricular tachycardia exit point in 25/27 (92\%) episodes with a mean timing of $-7.3 \pm 15.5 \mathrm{~ms}$ before QRS onset (range $0-50 \mathrm{~ms}$ ). The exit point was localized in all 21 post-myocardial infarction ventricular tachycardias, in all three arrhythmogenic right ventricular dysplasia ventricular tachycardias, but in only $1 / 3$ $(33 \%)$ idiopathic dilated cardiomyopathy ventricular tachycardias.

The diastolic pathway was identified in 19/27 (70\%) ventricular tachycardias. The earliest endocardial diastolic activity preceded the QRS onset by $-65 \cdot 3 \pm 49 \cdot 1 \mathrm{~ms}$ (range: -230 to $-25 \mathrm{~ms}$ ) corresponding to $38 \pm 23 \%$ (range $12-100 \%$ ) of the mean diastolic interval $(169 \cdot 25 \mathrm{~ms})$. The entire reentry circuit could be traced in only two ventricular tachycardias $(7 \%)$.

Among patients with a previous myocardial infarction, the diastolic pathway was mappable in 17/21 ventricular tachycardias $(80 \%)$, while it could be identified only in one arrhythmogenic right ventricular dysplasia ventricular tachycardia $(-35 \mathrm{~ms})$, and in one idiopathic dilated cardiomyopathy ventricular tachycardia $(-60 \mathrm{~ms})$. In 2/27 (both idiopathic dilated cardiomyopathy patients) ventricular tachycardias, neither the exit point nor the diastolic pathway could be identified.

\section{Ablation}

A total of 279 radiofrequency pulses were delivered, guided by off-line non-contact mapping analysis, to ablate 25 ventricular tachycardias. The mean number of radiofrequency pulses was $17 \cdot 4$ per patient (range: 5-39) and $11 \cdot 1$ per ventricular tachycardia (range 3-30).

Sixteen ventricular tachycardias $(64 \%)$ were successfully ablated. The success rate of ablation was higher when linear lesions were applied on the diastolic pathway (15/19 ventricular tachycardias, $78 \%)$ as compared to encircling lesions around the exit point (1/6 ventricular tachycardias 16\%) $(P=0.024)$ (Table 3). Ablation was not performed in two patients with idiopathic dilated cardiomyopathy (nos 5 and 12, Table 2) because of unclear mapping results without identification of any exit point and/or diastolic pathway of the only induced ventricular tachycardia. Catheter ablation was completely effective in 9/17 patients (53\%): 7/11 (63\%) of those with a prior myocardial infarction, $1 / 3(33 \%)$ with arrhythmogenic right ventricular dysplasia and $1 / 3$ $(33 \%)$ with idiopathic dilated cardiomyopathy. A partial success (ablation of one of two ventricular tachycardias) was obtained in one patient with a prior myocardial infarction. Ablation was not performed or it resulted unsuccessful in $7 / 17$ patients $(42 \%)$ (Table 3).

\section{Complications, procedure duration and radiation exposure}

No cardiac complication resulted from the use of the balloon catheter. One patient developed a haematoma at the site of placement of the arterial sheaths. One patient had a mild pericardial effusion, following catheter ablation, that resolved spontaneously within $24 \mathrm{~h}$. The mean total procedure time was $3.6 \pm 1 \mathrm{~h}$ (range: 1.8 to $5 \cdot 5$ ). The total fluoroscopy time averaged $36 \cdot 3 \pm 14 \mathrm{~min}$ (17-55 min) (Table 2). 


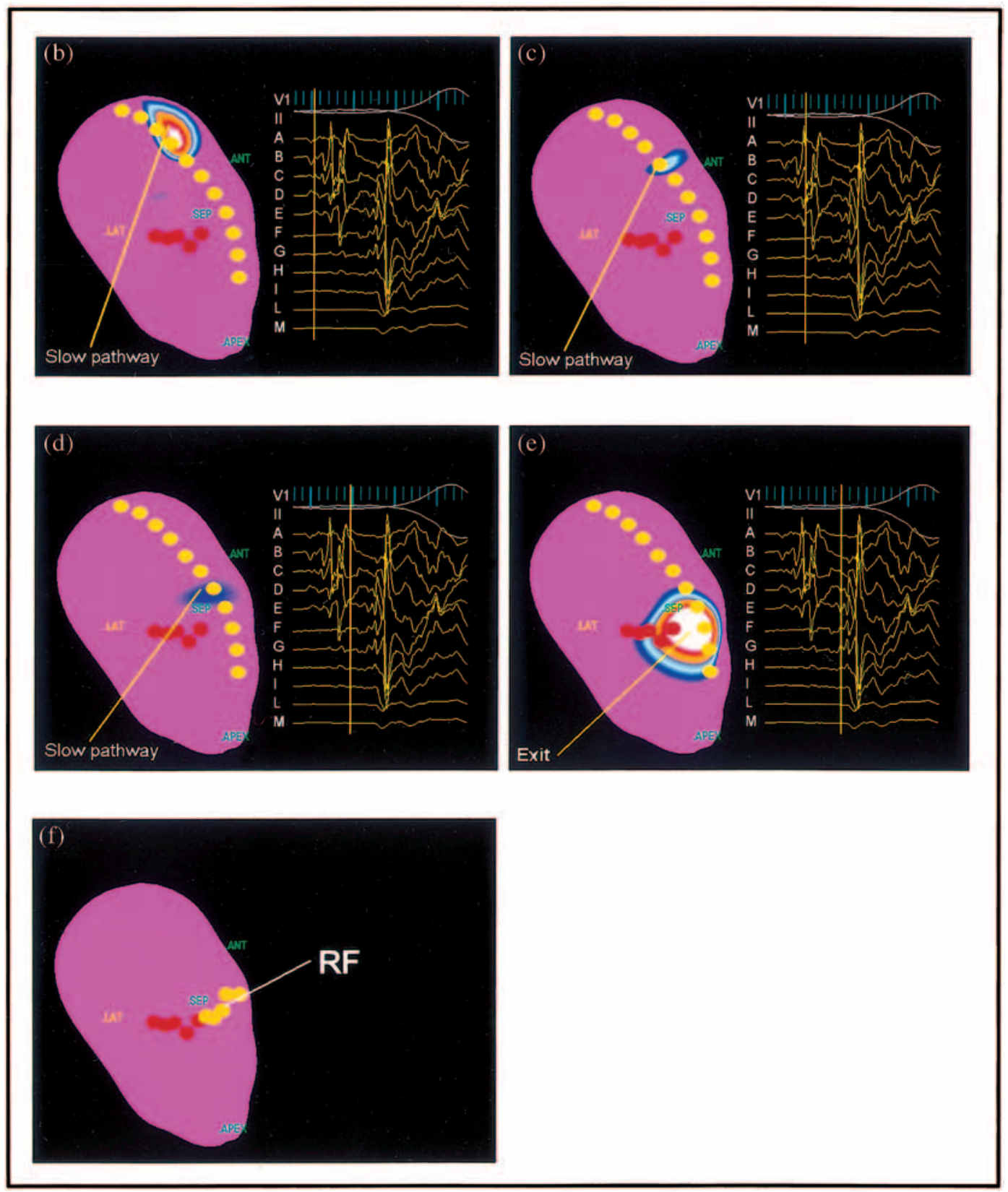

Figure 2 (b)-(f).

\section{Follow-up}

Follow-up (mean $20 \pm 5$ months; range 9-29 months) was available for all patients who had undergone mapping and ablation procedures. Ten patients $(59 \%)$ were free of any arrhythmia recurrence. There were no recurrences of the documented ventricular tachycardia in 10 patients in whom the arrhythmia had been successfully ablated; on the other hand, a high recurrence rate was observed after a failed procedure (5/7 patients, $71 \%)$ $(P<0 \cdot 01)$ (Table 3).

A new, previously non-documented ventricular tachycardia occurred in 2/10 (20\%) patients after a successful procedure and in $5 / 7$ patients after a failed procedure (71\%). Among four patients with a previously implanted ICD the mean number of ICD shocks decreased from $10 \pm 2 \cdot 5 /$ month to $0 \cdot 4 \pm 0 \cdot 2 /$ month $(P<0 \cdot 001)$. A new ICD was required in $6 / 13$ patients $(47 \%)$ following a failed (five patients) or partially successful (one patient) ablation. Two patients with a successful radiofrequency catheter ablation underwent ICD implant later during follow-up for a previously undocumented tolerated ventricular tachycardia recurrence.

Of the two patients implanted for arrhythmia recurrence after a successful procedure, one experienced ICD shocks. All five patients implanted after a failed 


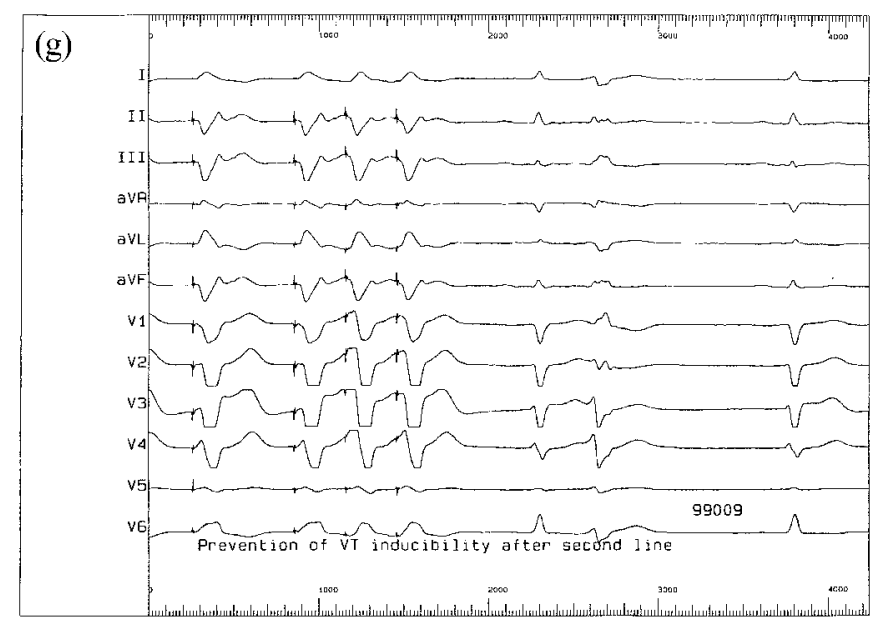

Figure 2 (g).

Figure 2 Panel a: Repeated programmed stimulation induced a ventricular tachycardia with almost identical morphology but longer cycle. Panels $b$ to f: Non-contact mapping revealed a similar pattern of diastolic activity with a shift of the diastolic pathway and the exit point toward the anterolateral wall. A second line of radiofrequency lesions (orange dots) aimed to cross the diastolic pathway before the exit was drawn during sinus rhythm. Panel g: Prevention of ventricular tachycardia reinducibility on programmed stimulation following second line.

radiofrequency catheter ablation had recurrence with ICD shocks. The patient implanted after partial radiofrequency catheter ablation success was free of arrhythmia recurrence and ICD therapies during follow-up. There were no deaths during the study.

\section{Discussion}

The feasibility of non-contact mapping to guide radiofrequency catheter ablation of human ventricular tachycardia has been demonstrated ${ }^{[15-17]}$; this, however, is the first study to test prospectively the efficacy of this mapping technique, in a consecutive series of patients presenting with clinical untolerated ventricular tachycardia inducible at electrophysiological study. As shown in the study, favourable acute and long-term results have been achieved in this selected group of patients.

\section{Non-contact mapping results}

The endocardial exit point of the ventricular tachycardia circuit was localized in all postinfarction ventricular tachycardias and in those related to right ventricular dysplasia, but only in one out of three ventricular tachycardias in idiopathic dilated cardiomyopathy, suggesting an intramural location of the reentry circuit in the remaining ventricular tachycardias in these patients. The identification of the exit site as a possible target is, however, of limited use, since the ablation in these cases is not usually effective: in our series only $1 / 6$ ventricular tachycardias, where radiofrequency current was delivered at the exit site, could be eliminated. Among patients with post-myocardial infarction ventricular tachycardia, a variable amount of the diastolic pathway could be traced in $17 / 21$ ventricular tachycardias $(80 \%)$, in keeping with published data ${ }^{[15-17]}$. The amount of the diastolic interval that could be traced on the virtual endocardium ranged from 12 to $100 \%$, averaging less than $40 \%$. This, however, fell in the range where successful ablation sites are found and concealed entrainment can be usually demonstrated ${ }^{[19,21,22]}$. The placement of linear lesions, by radiofrequency current, on the diastolic pathway resulted in successful ablation of $78 \%$ of targeted ventricular tachycardias in our series. The rationale of the ablation design was to create a line of block extending across the diastolic pathway thus preventing sustained reentry. The following limitation to this approach must be acknowledged:

(1) Since ablation was performed during sinus rhythm, termination of the ongoing ventricular tachycardia could not be assessed as a proof of success.

(2) The creation of bidirectional block along the diastolic pathway was not assessed, due to the impossibility of defining anatomical barriers by the non-contact mapping technique.

The entire ventricular tachycardia reentry circuit could be mapped endocardially in only two ventricular tachycardias $(8 \%)$ in two different patients. A slightly higher rate $(20 \%)$ of complete mapping has been reported by Schilling et al..$^{[15,16]}$, but only in $1 / 19$ ventricular 
Table 2 Procedural parameters

\begin{tabular}{|c|c|c|c|c|c|c|c|c|c|c|c|c|}
\hline Pt no. & VT no. & $\begin{array}{l}\mathrm{CL} \\
(\mathrm{ms})\end{array}$ & $\begin{array}{l}\mathrm{AP}^{*} 5 \mathrm{~s} \text { after } \\
\text { induction } \\
(\mathrm{mmHg})\end{array}$ & $\begin{array}{l}\text { AP* } 30 \mathrm{~s} \text { after } \\
\text { induction } \\
\text { (mmHg) }\end{array}$ & $\begin{array}{c}\text { EP } \\
\text { timing } \\
(\mathrm{ms})\end{array}$ & $\begin{array}{c}\text { DP } \\
\text { timing } \\
(\mathrm{ms})\end{array}$ & $\begin{array}{c}\text { DI } \\
(\mathrm{ms})\end{array}$ & $\begin{array}{l}\mathrm{RF} \\
\text { lesion }\end{array}$ & $\begin{array}{l}\text { No. of } \\
\text { RF }\end{array}$ & RF efficacy & $\begin{array}{l}\text { Procedure } \\
\text { (h) }\end{array}$ & $\begin{array}{l}\text { Fluoroscopy } \\
\text { (min) }\end{array}$ \\
\hline 1 & & 330 & 30 & 45 & 0 & -140 & 170 & $\mathrm{~L}$ & 10 & Y & $3 \cdot 5$ & $50 \cdot 0$ \\
\hline 2 & & 250 & 30 & 35 & 0 & -60 & 100 & $\mathrm{~L}$ & 8 & $\mathrm{Y}$ & $3 \cdot 0$ & $42 \cdot 0$ \\
\hline \multirow[t]{2}{*}{3} & I & 430 & 40 & 48 & -28 & -40 & 270 & $\mathrm{~L}$ & 10 & $\mathrm{~N}$ & $4 \cdot 2$ & $55 \cdot 0$ \\
\hline & II & 400 & 42 & 45 & 0 & -30 & 220 & $\mathrm{~L}$ & 10 & $\mathrm{~N}$ & & \\
\hline \multirow[t]{2}{*}{4} & I & 276 & 28 & 32 & -50 & -77 & 135 & $\mathrm{~L}$ & 15 & Y & $4 \cdot 5$ & $56 \cdot 0$ \\
\hline & II & 336 & 32 & 38 & -45 & -70 & 180 & $\mathrm{~L}$ & 10 & $\mathrm{Y}$ & & \\
\hline 5 & & 335 & 34 & 42 & $\mathrm{~N}$ & $\mathrm{~N}$ & 200 & NP & - & NP & $1 \cdot 8$ & $18 \cdot 0$ \\
\hline \multirow[t]{3}{*}{6} & I & 330 & 28 & 44 & 0 & -25 & 140 & $\mathrm{~L}$ & 12 & $\mathrm{Y}$ & $5 \cdot 2$ & $17 \cdot 0$ \\
\hline & II & 370 & 30 & 49 & -30 & -230 & 230 & L & 10 & $\mathrm{Y}$ & & \\
\hline & III & 360 & 30 & 46 & 0 & -150 & 170 & $\mathrm{~L}$ & 10 & Y & & \\
\hline \multirow[t]{3}{*}{7} & I & 340 & 35 & 54 & 0 & -55 & 180 & $\mathrm{~L}$ & 15 & $\mathrm{~N}$ & $5 \cdot 5$ & $38 \cdot 0$ \\
\hline & II & 290 & 32 & 46 & 0 & $\mathrm{~N}$ & 130 & E & 9 & $\mathrm{~N}$ & & \\
\hline & III & 255 & 30 & 40 & 0 & $\mathrm{~N}$ & 120 & E & 10 & $\mathrm{~N}$ & & \\
\hline 8 & & 260 & 40 & 55 & 0 & $\mathrm{~N}$ & 120 & E & 30 & $\mathrm{~N}$ & $4 \cdot 1$ & $40 \cdot 0$ \\
\hline 9 & & 250 & 42 & 48 & 0 & -35 & 120 & $\mathrm{~L}$ & 11 & $\mathrm{Y}$ & $3 \cdot 0$ & $25 \cdot 0$ \\
\hline \multirow[t]{2}{*}{10} & I & 410 & 44 & 50 & 0 & -30 & 240 & $\mathrm{~L}$ & 6 & $\mathrm{Y}$ & $4 \cdot 0$ & $33 \cdot 0$ \\
\hline & II & 390 & 42 & 48 & 0 & -80 & 220 & $\mathrm{~L}$ & 15 & $\mathrm{~N}$ & & \\
\hline \multirow[t]{2}{*}{11} & I & 450 & 38 & 49 & 0 & -85 & 300 & $\mathrm{~L}$ & 3 & $\mathrm{Y}$ & $3 \cdot 2$ & $56 \cdot 0$ \\
\hline & II & 315 & 34 & 42 & 0 & -40 & 155 & $\mathrm{~L}$ & 5 & $\mathrm{Y}$ & & \\
\hline 12 & & 280 & 40 & 46 & $\mathrm{~N}$ & $\mathrm{~N}$ & 140 & NP & - & NP & $2 \cdot 0$ & $20 \cdot 0$ \\
\hline 13 & & 270 & 42 & 48 & -30 & $\mathrm{~N}$ & 140 & $\mathrm{E}$ & 5 & $\mathrm{~N}$ & $2 \cdot 8$ & $20 \cdot 0$ \\
\hline \multirow[t]{3}{*}{14} & I & 280 & 29 & 35 & 0 & -45 & 140 & $\mathrm{~L}$ & 15 & Y & $4 \cdot 0$ & $46 \cdot 0$ \\
\hline & II & 340 & 31 & 42 & 0 & -40 & 200 & $\mathrm{~L}$ & 8 & $\mathrm{Y}$ & & \\
\hline & III & 350 & 30 & 48 & 0 & $\mathrm{~N}$ & 200 & $\mathrm{E}$ & 6 & $\mathrm{Y}$ & & \\
\hline 15 & & 250 & 28 & 36 & 0 & -60 & 110 & $\mathrm{~L}$ & 20 & $\mathrm{Y}$ & $3 \cdot 5$ & $29 \cdot 0$ \\
\hline 16 & & 230 & 29 & 44 & 0 & -50 & 110 & $\mathrm{~L}$ & 12 & $\mathrm{Y}$ & $3 \cdot 0$ & $35 \cdot 0$ \\
\hline 17 & & 280 & 41 & 50 & 0 & $\mathrm{~N}$ & 130 & E & 14 & $\mathrm{~N}$ & $3 \cdot 0$ & $32 \cdot 0$ \\
\hline Mean & & $320 \cdot 63$ & $34 \cdot 4$ & $44 \cdot 6$ & $-7 \cdot 3$ & $-65 \cdot 30$ & $169 \cdot 26$ & & $11 \cdot 1$ & & $3 \cdot 5$ & $36 \cdot 0$ \\
\hline DS & & $60 \cdot 66$ & $5 \cdot 53$ & $5 \cdot 75$ & $15 \cdot 5$ & $49 \cdot 1$ & $52 \cdot 31$ & & $5 \cdot 52$ & & $1 \cdot 0$ & $13 \cdot 4$ \\
\hline
\end{tabular}

$\mathrm{CL}=$ cycle length; $\mathrm{EP}=$ exit point; $\mathrm{DP}=$ diastolic pathway; $\mathrm{DI}=$ diastolic interval; $\mathrm{L}=$ linear lesion across diastolic pathway; $\mathrm{E}=$ encircling lesion on the exit point; $\mathrm{NP}=$ not performed; $\mathrm{Y}=$ yes; $\mathrm{N}=$ no.

*Mean arterial pressure.

Table 3 Ablation results and follow-up

\begin{tabular}{|c|c|c|c|c|c|c|c|}
\hline \multicolumn{4}{|c|}{ Ventricular tachycardia data } & \multicolumn{4}{|c|}{ Patient data } \\
\hline $\begin{array}{l}\mathrm{RFCA} \text { on } \\
\text { target VT }\end{array}$ & $\begin{array}{l}\text { No. of } \\
\text { target VTs }\end{array}$ & On EP & On DP & $\begin{array}{l}\text { RFCA } \\
\text { result }\end{array}$ & $\begin{array}{l}\text { No. of } \\
\text { patients }\end{array}$ & $\begin{array}{c}\text { Any VT recurrence } \\
\text { (no. of patients and \%) }\end{array}$ & $\begin{array}{l}\text { Target VT recurrence } \\
\text { (no. of patients and } \% \text { ) }\end{array}$ \\
\hline Success & $16(64 \%)$ & $1(16 \%)^{*}$ & $15(78 \%)^{*}$ & $\begin{array}{l}\text { Full success } \\
\text { Partial success }\end{array}$ & $\begin{array}{c}9(54 \%) \\
1(5 \%)\end{array}$ & $\begin{array}{c}2 / 9(22 \%) \\
0 / 1(0 \%)\end{array}$ & $\begin{array}{l}0 / 9(0 \%) \\
0 / 1(0 \%)\end{array}$ \\
\hline Failure & $9(36 \%)$ & $5(74 \%)$ & $4(22 \%)$ & Failure ${ }^{* *}$ & $7(41 \%)^{* *}$ & $5 / 7(71 \%)$ & $5 / 7(71 \%)^{* * *}$ \\
\hline Total & $25^{\circ}$ & 6 & 19 & Total & 17 & $7 / 17(41 \%)$ & $4 / 17(23 \%)$ \\
\hline
\end{tabular}

$\mathrm{EP}=$ exit point; $\mathrm{DP}=$ diastolic pathway.

${ }^{\circ}$ Two ventricular tachycardias in two idiopathic dilated cardiomyopathy patients with inconclusive mapping were not targeted for radiofrequency catheter ablation.

$* P=0 \cdot 062$.

**Includes five patients with target ventricular tachycardia reinducible after radiofrequency catheter ablation and two idiopathic dilated cardiomyopathy patients with inconclusive mapping.

$* * * P<0 \cdot 01$ vs radiofrequency catheter ablation success.

tachycardias in the study of Strickberger et al. ${ }^{[17]}$. This reflects the fact that only in a minority of cases is the reentry circuit entirely confined to the endocardial surface, due to the presence of intramural or subepicardial areas of slow conduction, as shown by surgical mapping data $^{[23]}$
On the other hand, the accurate identification of diastolic signals may be limited:

- Very low amplitude signals may not be detected, particularly if the distance between the centre of the balloon catheter and endocardial surface exceeds $40 \mathrm{~mm}$. 
- Since the geometry of the left ventricle is contoured at the beginning of the study during sinus rhythm, changes of the ventricular size and of the contraction pattern during ventricular tachycardia may adversely affect the accuracy of the location of the endocardial electrograms.

In spite of these theoretical limitations, identification of areas of endocardium critical to the maintenance of the reentry circuit allowed an effective ablation of $65 \%$ of ventricular tachycardias with complete prevention of arrhythmia recurrence in the seven of the nine patients who were rendered non-inducible after radiofrequency catheter ablation, and in one patient in whom the ablation was only partially effective.

\section{Ablation during sinus rhythm: comparison with other techniques}

The feasibility of catheter ablation of untolerated ventricular tachycardia is related to the possibility of performing the procedure during sinus rhythm. The identification of suitable target sites has been directed by the search for fragmented low amplitude potentials and/or sites with the latest activation with respect to the surface QRS; although these characteristics are typically associated with a successful ablation site, they can also be demonstrated at sites not related to the ventricular tachycardia circuit and the specificity of fragmented and low-amplitude electrograms during sinus rhythm in localizing the ventricular tachycardia ablation site is very low ${ }^{[24-27]}$. According to a non-contact mapping study, only $30 \%$ of the sites displaying the latest activation during sinus rhythm are located within $2 \mathrm{~cm}$ of the actual successful ablation site in ischaemic ventricular tachycardia ${ }^{[13]}$. In two recent studies ${ }^{[28,29]}$, patients with untolerated ventricular tachycardia were treated by radiofrequency catheter ablation performed during sinus rhythm and guided by pacemapping plus detection of fragmented electrograms and/or scar site with favourable short and long-term results.

A more precise location of the scar and of strands of surviving myocardium can be achieved by electroanatomic mapping. This technique guides the deployment of ablation lesion lines from scarred to healthy myocardium, aimed at interrupting the hypothetical reentry circuit. In a recent study ${ }^{[30]}$, prevention of ventricular tachycardia inducibility was achieved in seven out of 15 patients $(46 \%)$.

Similar acute and long-term results were achieved by non-contact mapping in this series, although with an overall shorter procedure time and fewer radiofrequency lesions per ventricular tachycardia and per patient. This difference can be explained by the more selective type of intervention.

\section{Conclusions}

Non-contact mapping was used to guide radiofrequency catheter ablation in 17/22 consecutive patients with untolerated ventricular tachycardia. Given the favourable clinical long-term results, the use of non-contact mapping offers a significant advantage when considering catheter ablation of untolerated ventricular tachycardia. This approach appears to be more effective in postmyocardial infarction patients, due to the likelihood of the more stable anatomy of the reentry circuit, in comparison with patients with non-ischaemic cardiomyopathy.

Although there were no recurrences of the successfully ablated ventricular tachycardias, arrhythmic events due to previous not documented or non-inducible ventricular tachycardias occurred later during follow-up in 2/9 patients $(22 \%)$ (one with post-myocardial infarction ventricular tachycardia and one with idiopathic dilated cardiomyopathy) after a fully successful radiofrequency catheter ablation. No statement therefore can be made on the long-term safety of catheter ablation as a primary treatment of untolerated ventricular tachycardia without considering a subsequent ICD placement.

\section{References}

[1] Morady F, Frank R, Kou WH et al. Identification and catheter ablation of slow conduction in the reentrant circuit of ventricular tachycardia in humans. J Am Coll Cardiol 1988; 11: $775-82$

[2] Fontaine G, Frank R, Tonet JL, Grosgogeat Y. Identification of zones of slow conduction appropriate for VT ablation: theoretical and practical considerations. Pacing Clin Electrophysiol 1998; 12: 262-7.

[3] Morady F, Kadish A, Rosenbeek S et al. Concealed entrainment as a guide for catheter ablation of ventricular tachycardia in patients with prior myocardial infarction. J Am Coll Cardiol 1991; 17: 678-89.

[4] Stevenson WG, Khan H, Sager P et al. Identification of reentry circuit sites during catheter mapping and radiofrequency ablation of ventricular tachycardia late after myocardial infarction. Circulation 1993; 88: 1647-70.

[5] Kim YH, Sosa-Suarez G, Trouton TG et al. Treatment of ventricular tachycardia by transcatheter radiofrequency ablation in patients with ischemic heart disease. Circulation 1994; 89: 1094-1102.

[6] Stevenson WG, Friedman PL, Sager PT et al. Exploring postinfarction reentrant ventricular tachycardia with entrainment mapping. J Am Coll Cardiol 1997; 29: 1180-9.

[7] Stevenson WG, Sager PT, Friedman PL. Entrainment techniques for mapping atrial and ventricular tachycardias. J Cardiovasc Electrophysiol 1995; 6: 201-16.

[8] Bogun F, Bahu M, Knight P et al. Comparison of effective and ineffective target sites that demonstrate concealed entrainment in patients with coronary artery disease undergoing radiofrequency ablation of ventricular tachycardia. Circulation 1997; 95: 183-90.

[9] Morady F, Harvey M, Kalbfleisch SJ, El-Atassi R, Calkins H, Langberg JJ. Radiofrequency catheter ablation of ventricular tachycardia in patients with coronary artery disease. Circulation 1993; 87: 363-72.

[10] Stevenson WG, Friedman PL, Sweeney MO. Catheter ablation as an adjunct to ICD therapy. Circulation 1997; 96: 1378-80.

[11] Peters NS, Jackman W, Schilling RJ, Beatty G, Davies DW. Human left ventricular endocardial activation mapping using a novel non-contact catheter. Circulation 1997; 95: 1658-60.

[12] Schilling RJ, Peters NS, Davies DW. Simultaneous endocardial mapping in the human left ventricle using a non-contact 
catheter: comparison of contact and reconstructed electrograms during sinus rhythm. Circulation 1998; 98: 887-98.

[13] Schilling RJ, Davies DW, Peters NS. Characteristics of sinus rhythm electrograms at sites of ablation of ventricular tachycardia relative to all other sites: a non-contact mapping study of the entire left ventricle. J Cardiovasc Electrophysiol 1998; 9: 921-33.

[14] Gornik CG, Adler SW, Pederson BA, Hauck J, Budd J, Schweitzer J. Validation of a new non-contact catheter system for electroanatomic mapping of left ventricular endocardium. Circulation 1999; 99: 829-35.

[15] Schilling RJ, Peters NS, Davies DW. Feasibility of a noncontact catheter for endocardial mapping of human ventricular tachycardia. Circulation 1999; 99: 2543-52.

[16] Schilling RJ, Peters NS, Davies DW. Mapping and ablation of ventricular tachycardia with the aid of a non-contact mapping system. Heart 1999; 81: 570-5.

[17] Strickberger SA, Knight BP, Michaud GF, Pelosi F, Morady F. Mapping and ablation of ventricular tachycardia guided by virtual electrograms using a non-contact, computerized mapping system. J Am Coll Cardiol 2000; 35: 414-21.

[18] Downar E, Harris L, Mickleborough LL, Shaikh N, Parson ID. Endocardial mapping of ventricular tachycardia in the intact human ventricle: Evidence of reentrant mechanisms. J Am Coll Cardiol 1988; 11: 783-91.

[19] de Bakker JM, van Capelle FJ, Janse MJ et al. Macroreentry in the infarcted human heart: the mechanism of ventricular tachycardia with a focal activation pattern. J Am Coll Cardiol 1981; 18: 1005-14.

[20] Stevenson WG. Catheter mapping of ventricular tachycardia. In: Zipes DP, Jalife J, eds. Cardiac Electrophysiology: From Cell to Bedside, 2nd ed. Philadelphia: WB Saunders Company, 1995: 1093-12.

[21] Svenson RH, Littmann L, Colavita PG et al. Laser photoablation of ventricular tachycardia; correlation of diastolic activation times and photoablation effects on cycle length and termination-observation supporting a macroreentrant mechanism. J Am Coll Cardiol 1992; 19: 607-13.
[22] Cabo C, Schmitt H, Masters G, Coromilas J, Wit AL, Scheinman MM. Location of diastolic potentials in reentrant circuits causing sustained ventricular tachycardia in the infarcted canine heart. Circulation 1998; 98: 2598-2607.

[23] Kaltenbrunner W, Cardinal R, Dubuc M et al. Epicardial and endocardial mapping of ventricular tachycardia in patients with myocardial infarction. Is the origin of the tachycardia always subendocardially localized? Circulation 1991; 84: 1058-71.

[24] Stevenson WG, Weiss JN, Wiener I et al. Fractionated endocardial electrograms are associated with slow conduction in human: Evidence from pacemapping. J Am Coll Cardiol 1989; 13: 369-76.

[25] Miller JM, Tyson GS, Hargrove WC 3rd, Vassallo JA, Rosenthal ME, Josephson ME. Effect of subendocardial resection on sinus rhythm endocardial electrogram abnormalities. Circulation 1995; 92: 2385-91.

[26] Stevenson WG, Sager PT, Natterson PD, Saxon LA, Middlekauff HR, Wiener I. Relation of pace mapping QRS configuration and conduction delay to ventricular tachycardia reeentry circuits in human infarct scars. J Am Coll Cardiol 1995; 26: 481-8.

[27] Harada T, Stevenson WG, Kocovic DZ, Friedman PL. Catheter ablation of ventricular tachycardia after myocardial infarction: Relation of endocardial sinus rhythm late potentials to the reentry circuit. J Am Coll Cardiol 1997; 30: 1015-23.

[28] Ellison KE, Stevenson WG, Sweeney MO, Lefroy DC, Delacretaz E, Friedman PL. Catheter ablation for hemodynamically unstable monomorphic ventricular tachycardia. J Cardiovasc Electrophysiol 2000; 11: 41-4.

[29] Furniss S, Anil-Kumar R, Bourke JP, Behulova R, Simeonidou E. Radiofrequency ablation of haemodynamically unstable ventricular tachycardia after myocardial infarction. Heart 2000; 84: 648-52.

[30] Marchlinsky FE, Callans DJ, Gottlieb CD, Zado E. Linear ablation lesions for control of unmappable ventricular tachycardia in patients with ischemic and nonischemic cardiomyopathy. Circulation 2000; 101: 1288-96. 